\title{
Molecular genotyping of human Ureaplasma species based on multiple-banded antigen (MBA) gene sequences
}

\author{
Fanrong Kong, Zhenfang Ma, Gregory James, Susanna Gordon \\ and Gwendolyn L. Gilbert
}

Centre for Infectious

Diseases and Microbiology,

Institute of Clinical

Pathology and Medical

Research, Westmead

Hospital, Darcy Rd,

Westmead, New South

Wales, 2145 Australia
Author for correspondence: Gwendolyn L. Gilbert. Tel: +6129845 6255. Fax: +61298938659. e-mail: lyng@icpmr.wsahs.nsw.gov.au

Ureaplasma urealyticum has been divided into 14 serovars. Recently, subdivision of $U$. urealyticum into two species has been proposed: $U$. parvum (previously $U$. urealyticum parvo biovar), comprising four serovars $(1,3,6,14)$ and $U$. urealyticum (previously $U$. urealyticum T-960 biovar), 10 serovars (2, 4, 5, 7-13). The multiple-banded antigen (MBA) genes of these species contain both species and serovar/subtype specific sequences. Based on whole sequences of the 5'-ends of MBA genes of $U$. parvum serovars and partial sequences of the 5 '-ends of MBA genes of $U$. urealyticum serovars, we previously divided each of these species into three MBA genotypes. To further elucidate the relationships between serovars, we sequenced the whole 5'-ends of MBA genes of all 10 $U$. urealyticum serovars and partial repetitive regions of these genes from all serovars of $U$. parvum and $U$. urealyticum. For the first time, all four serovars of $U$. parvum were clearly differentiated from each other. In addition, the 10 serovars of $U$. urealyticum were divided into five MBA genotypes, as follows: MBA genotype A comprises serovars 2, 5, 8; MBA genotype B, serovar 10 only; MBA genotype $C$, serovars 4, 12, 13; MBA genotype $D$, serovar 9 only; and MBA genotype $E$ comprises serovars 7 and 11. There were no sequence differences between members within each MBA genotype. Further work is required to identify other genes or other regions of the MBA genes that may be used to differentiate $U$. urealyticum serovars within MBA genotypes $A, C$ and $E$. A better understanding of the molecular basis of serotype differentiation will help to improve subtyping methods for use in studies of the pathogenesis and epidemiology of these organisms.

Keywords: Ureaplasma parvum, Ureaplasma urealyticum, MBA gene, subtyping

\section{INTRODUCTION}

Human ureaplasmas are recognized causes of urethritis (Hewish et al., 1986; Cracea et al., 1985; TaylorRobinson et al., 1985), and have been associated with complications of pregnancy and prematurity (AbeleHorn et al., 1997; Robertson et al., 1986; Kundsin et al., 1996; Hannaford et al., 1999). However, as common genital tract commensals (Viarengo et al., 1980; Cracea et al., 1985), their pathogenic roles in individual cases are difficult to confirm (Zheng et al., 1992).

Abbreviation: MBA, multiple-banded antigen.
The former species Ureaplasma urealyticum contained 14 serovars (Razin \& Yogev, 1986; Robertson \& Stemke, 1982). In the proposed new taxonomy, Ureaplasma parvum (previously $U$. urealyticum parvo biovar), contains four and U. urealyticum (previously U. urealyticum T-960 biovar), 10 serovars (Kong et al., 1999b). The relationship between serovars and disease syndromes needs to be studied further (Grattard et al., 1995; Naessens et al., 1988). However, this has been limited by technical difficulties and cross-reactions associated with serotyping (Wiley \& Quinn, 1984; Quinn et al., 1981; Robertson \& Stemke, 1979; Stemke \& Robertson, 1985), even when monoclonal antibodies were used (Naessens et al., 1998).

Better understanding of the genetic basis of the 
Table 1. Oligonucleotide primers used in this study

\begin{tabular}{|c|c|c|c|}
\hline Primer & Specificity & $T_{\mathrm{m}}\left({ }^{\circ} \mathbf{C}\right)$ & Sequence \\
\hline UMS-125 & Ureaplasma spp. & 66 & -151GTA TTT GCA ATC TTT ATA TGT TTT CG-125 \\
\hline UMS-57 & U. parvum & 66 & -84(A/G)(A/C)(T/C)AA ATC TTA GTG TTC ATA TTT TTT AC-57 \\
\hline UMSPS1* & U. parvum & 68 & 319CCT CGT GAA CCA AAA CCT AAT G340 \\
\hline UMSPS2* & U. parvum & 66 & 367GGA TTA ATC AAG ACT TCA GGT TTG390 \\
\hline UMA1213 & UP $3 / 14$ & 68 & 1239CTA AAG TAA TTA TTT TCC AGT AGT TTC1213 \\
\hline UMA1586 & UP $3 / 14$ & 72 & 1613GAT AAT CAT TCA TCT TCT CTT AAT TGT C1586 \\
\hline UMS3S* & UP 3 & 68 & -107TTA CTG TAG AAA TTA TGT AAG ATT ACC-81 \\
\hline UMS14S* & UP 14 & 68 & -109AAT TAC TGT AGA AAT TAT GTA AGA TTA AT-81 \\
\hline UMA269 & UP $3 / 14$ & 66 & 293AA CTA AAT GAC CTT TTT CAA GTG TAC269 \\
\hline UMA314A* & UP $3 / 14$ & 68 & 463GTT GTT CTT TAC CTG GTT GTG TAG440 \\
\hline UMA $314 A^{\prime *}$ & UP $3 / 14$ & 68 & 465TG/TG TTG TTC TTT ACC TGG TTG TGT A441 \\
\hline UMS-54 & UP 6 & 66 & -77AAT CTT AGT GTT CAT ATT TTT TAC TAG-54 \\
\hline UMA6A* & UP 6 & 66 & 464CCT GGT TCT TGA GTT TTC GGA G443 \\
\hline UMA6A $*$ & UP 6 & 68 & 468TTT ACC TGG TTC TTG AGT TTT CGG445 \\
\hline UMS-83 & UP 1 & 66 & -107TTACT GTA GAA ATT ATG TAA GAT TGC-83 \\
\hline UMA1A* & UP 1 & 70 & 469TTT CTT TTG GTT CTT CAG TTT TTG AAG443 \\
\hline UMA1A ${ }^{\prime *}$ & UP 1 & 68 & 471ATT TTC TTT TGG TTC TTC AGT TTT TGA445 \\
\hline UMA269' & UP $1 / 6$ & 66 & 293ACCA AAT GAC CTT TTG TAA CTA GAT269 \\
\hline UMS-170 & Ureaplasma spp. & 66 & -195GTA TTT GCA ATC TTT ATA TGT TTT CG-170 \\
\hline UMS-61 & U. urealyticum & 66 & -83TATA TTT GCA AAA CTA TAA ATA GAC AC-61 \\
\hline UMSUS* & U. urealyticum & 66 & 163GTT TAC GAC ATT GAA AAT TTC GAT G187 \\
\hline UMAUA* & U. urealyticum & 62 & 466GGG $(\mathrm{G} / \mathrm{T})(\mathrm{A} / \mathrm{T}) \mathrm{G} \mathrm{TT}(\mathrm{G} / \mathrm{T})(\mathrm{A} / \mathrm{C} / \mathrm{T}) \mathrm{AC} \mathrm{CA}(\mathrm{C} / \mathrm{T}) \mathrm{T}(\mathrm{G} / \mathrm{T}) \mathrm{C}$ CTG GTT443 \\
\hline UMSUS1* & U. urealyticum & 62 & 378AAC TGC ATC T(C/T)T AG(C/T) ATT ACC T399 \\
\hline UMSUS2* & U. urealyticum & 62 & 397CCT GAT AAT TT(G/T) AAT TAT CAA ACA G421 \\
\hline UMAUA1* & U. urealyticum & 66 & 1537GCC CAA TTC ATA GGC TAT TAA TTG1514 \\
\hline UMAUA2* & U. urealyticum & 64 & 1546AAA AAA ATA GCC CAA TTC ATA GGC1523 \\
\hline UMA2A $1 *$ & $\mathrm{UU} \mathrm{A} / \mathrm{B}$ & 66 & 451TTC CTG GTT TTG TTT CAA AAC CTA T427 \\
\hline UMA2A $2 *$ & $\mathrm{UU}$ A/B & 66 & 454CAC TTC CTG GTT TTG TTT CAA AAC431 \\
\hline UMA2A* & UU A & 66 & 479CCA CTT CCT GGT TTT GTA GTT TC457 \\
\hline UMA10A* & UU B & 68 & 479CCA CTT CCT GGT TGT GTA GTT GA457 \\
\hline UMA4A $1 *$ & UU C & 68 & 452TT GCC TGG TTG TGT TTC GAA CTC430 \\
\hline UMA4A2* & UU C & 68 & 454CAT TGC CTG GTT GTG TTT CGA AC432 \\
\hline UMA9A $1 *$ & UU D & 66 & 460CTG GAG TTG GTG TAG GCG CAT440 \\
\hline UMA9A $2 *$ & UU D & 66 & 462TTC TGG AGT TGG TGT AGG CGC442 \\
\hline UMA7A1 & UU E & 74 & 245GTA ATT GCA ACA TGG AAT TCA GTT TCA219 \\
\hline UMA7A $2 *$ & UU E & 66 & 458GGT TCT GGT GTA TGA GTG CTT TT436 \\
\hline UMA7A $3 *$ & UU E & 66 & 461GTT GGT TCT GGT GTA TGA GTG C440 \\
\hline
\end{tabular}

* Primers designed specifically for this study. All others have been previously published (see text for references).

The melting temperatures $\left(T_{\mathrm{m}}\right)$ of primers were calculated by the formula: $T_{\mathrm{m}}=4 \times$ no. of $(\mathrm{G}+\mathrm{C})+2 \times$ no. of $(\mathrm{A}+\mathrm{T})$.

conventional ureaplasma serotype classification will assist in development of a practical molecular serotyping system and allow further investigation of the pathogenic potential of individual subtypes/serovars (Robertson \& Stemke, 1982; Kong et al., 1999a). In our previous study, we sequenced three genes and adjoining regions of all 14 ureaplasma serovars and studied the phylogenetic relationships between them (Kong et al., 1999b). We showed that the sequences of the $16 \mathrm{~S}$ rRNA genes and $16 \mathrm{~S}-23 \mathrm{~S}$ rRNA intergenic spacer regions, the urease gene subunits ure $A$, ure $B$, partial ure $C$ and adjoining regions - upstream of ure $A$, ure $A$-ure $B$ spacer, and the ure $B-$ ure $C$ spacer - were generally conserved for serovars within each of the two proposed new species. Only the $5^{\prime}$-end regions of the MBA genes showed heterogeneity between the four serovars of $U$. parvum and the 10 serovars of $U$. urealyticum.

It has been suggested, previously, that the repetitive region of the MBA gene would contain serovar-specific sites (Watson et al., 1990; Zheng et al., 1996). In this study, we sequenced partial repetitive regions of the MBA genes of all 14 ureaplasma serovars, to determine whether these genes could provide further evidence to support the present serotype classification and improve our previous molecular subtyping system for $U$. parvum and U. urealyticum (Kong et al., 1999a, 2000). 


\section{METHODS}

Bacterial strains. Two sets of reference strains of all 14 serovars of $U$. parvum and $U$. urealyticum were used as previously described (Kong et al., 1999b). One set was obtained directly from the American Type Culture Collection (ATCC reference strains) and the other was kindly provided by Dr H. L. Watson, Department of Microbiology, University of Alabama at Birmingham, AL, USA (UAB reference strains).

Oligonucleotide primers. The oligonucleotide primers used in this study are shown in Table 1. Previously published oligonucleotide primers UMS-125, UMA1213, UMA1586 (Zheng et al., 1995), UMS-57 (Kong et al., 2000), and new primers designed by us - UMSPS1, UMSPS2, UMAUA based on previously published sequences (Zheng et al., 1999; GenBank accession nos U50459, U50460, U50461) were used to sequence the repetitive regions of the MBA genes of the four $U$. parvum serovars. Previously published oligonucleotide primers UMS-170 (Teng et al., 1995), UMS-61 (Kong et al., 2000), and new primers designed by us UMSUS, UMSUS1, UMSUS2, UMAUA, UMAUA1, UMAUA2 - based on the previously published sequences (Kong et al., 1999b; Zheng et al., 1999; GenBank accession nos U50459, U50460, U50461) were used to sequence the 5'end and the repetitive regions of the MBA genes of all the 10 $U$. urealyticum serovars.

Additional new primers-UMS3S, UMA314A, UMA314A', UMS14S, UMA1A, UMA1A', UMA6A, $\mathrm{UMA}^{\prime} \mathrm{A}^{\prime}$ - based on sequences determined in this and previous studies (Kong et al., 1999b), and previously published primers designed by us UMS-83, UMS-54, UMA269 and UMA269' were designed specifically to amplify and differentiate MBA genes of four $U$. parvum serovars 3, 14, 1 and 6. New primers UMA2A1, UMA2A2; UMA2A; UMA10A; UMA4A1, UMA4A2; UMA9A1, UMA9A2; UMA7A2 and UMA7A3, based on the sequences obtained in this study, and the previously published primer UMA7A1 (Kong et al., 2000) were designed to amplify and differentiate MBA genotypes A/B, MBA genotype $\mathrm{A}$, MBA genotype $\mathrm{B}$, MBA genotype $\mathrm{C}$, MBA genotype D and MBA genotype $\mathrm{E}$ (Table 1).

DNA preparations and PCR. DNA preparations and PCR systems were used as previously described (Kong et al., 1999a, b). To amplify the repetitive regions of the MBA genes of $U$. parvum serovars for sequencing, a nested PCR was developed, using UMS-125/UMA1586 as outer primers and UMS-57/UMA1213 (for serovars 3 and 14) and UMS-57/UMAUA (for all four serovars of $U$. parvum) as inner primers. Nested PCR was also used to amplify the $5^{\prime}$-ends and repetitive regions of the MBA genes of the $10 \mathrm{U}$. urealyticum serovars for sequencing. The outer primers were UMS-170/UMAUA2 and inner primers were UMS-61/UMAUA and UMSUS/UMAUA2 (or UMSUS/UMAUA1) (Table 1).

The denaturation, annealing and elongation temperatures and times used for the first step PCR were $95^{\circ} \mathrm{C}$ for $30 \mathrm{~s}$, $50{ }^{\circ} \mathrm{C}$ for $30 \mathrm{~s}$ and $72^{\circ} \mathrm{C}$ for $3 \mathrm{~min}$, respectively, for 30 cycles. For the second step PCR, the denaturation, annealing and elongation temperatures and times used were $95^{\circ} \mathrm{C}$ for $30 \mathrm{~s}$, $55^{\circ} \mathrm{C}$ for $30 \mathrm{~s}$ and $72^{\circ} \mathrm{C}$ for $2 \mathrm{~min}$, for 30 cycles. For the serovar-/MBA genotype-specific PCR, the denaturation, annealing and elongation temperatures and times used were $95^{\circ} \mathrm{C}$ for $30 \mathrm{~s}, 55-62{ }^{\circ} \mathrm{C}$ (according to the $T_{\mathrm{m}}$ value) for $30 \mathrm{~s}$ and $72{ }^{\circ} \mathrm{C}$ for $1 \mathrm{~min}$, respectively, for 40 cycles.
Table 2. Primer pairs used to differentiate all 14 serovars of $U$. parvum and $U$. urealyticum and summary of PCR results showing sizes of bands (amplicons)

See Table 1 for primer sequences. UP 3: U. parvum serovar 3; UP 14: $U$. parvum serovar 14; UP 1: U. parvum serovar 1; UP 6: U. parvum serovar 6. UU A/B: U. urealyticum MBA genotypes $\mathrm{A}$ and $\mathrm{B}$, includes serovars 2, 5, 8 and 10; UU A: $U$. urealyticum MBA genotype $\mathrm{A}$, includes serovars 2,5 and 8; UU B: U. urealyticum MBA genotype B, includes serovar 10; UU C: $U$. urealyticum MBA genotype $\mathrm{C}$, includes serovars 4, 12 and 13; UU D: U. urealyticum MBA genotype D, includes serovar 9; UU E: $U$. urealyticum MBA genotype E, includes serovars 7 and 11. See Figs 4 and 5 for PCR results for UMS-83 UMA269' and UMS-61 UMA7A1, respectively.

\begin{tabular}{|llc|}
\hline Primer pairs & $\begin{array}{c}\text { Specificity } \\
\text { (subtype/serovar) }\end{array}$ & $\begin{array}{c}\text { Amplicon size } \\
\text { (bp) }\end{array}$ \\
\hline UMS3S UMA269 & UP 3 & 400 \\
UMS3S UMA314A & UP 3 & 570 \\
UMS3S UMA314A' & UP 3 & 572 \\
UMS14S UMA269 & UP 14 & 402 \\
UMS14S UMA314A & UP 14 & 572 \\
UMS14S UMA314A' & UP 14 & 574 \\
UMS-83 UMA1A & UP 1 & 576 \\
UMS-83 UMA1A' & UP 1 & 578 \\
UMS-83 UMA269' & UP 1 & 400 \\
UMS-54 UMA6A & UP 6 & 544 \\
UMS-54 UMA6A' & UP 6 & 548 \\
UMS-54 UMA269' & UP 6 & 370 \\
UMS-61 UMA2A1 & UU A/B & 539 \\
UMSUS UMA2A1 & UU A/B & 289 \\
UMS-61 UMA2A2 & UU A/B & 537 \\
UMSUS UMA2A2 & UU A/B & 292 \\
UMS-61 UMA2A & UU A & 562 \\
UMSUS UMA2A & UU A & 317 \\
UMS-61 UMA10A & UU B & 562 \\
UMSUS UMA10A & UU B & 317 \\
UMS-61 UMA4A1 & UU C & 535 \\
UMSUS UMA4A1 & UU C & 290 \\
UMS-61 UMA4A2 & UU C & 537 \\
UMSUS UMA4A2 & UU C & 292 \\
UMS-61 UMA9A1 & UU D & 543 \\
UMSUS UMA9A1 & UU D & 298 \\
UMS-61 UMA9A2 & UU D & 545 \\
UMSUS UMA9A2 & UU D & 300 \\
UMS-61 UMA7A1 & UU E & 328 \\
UMS-61 UMA7A2 & UU E & 541 \\
UMSUS UMA7A2 & UU E & 296 \\
UMS-61 UMA7A3 & UU E & 544 \\
UMSUS UMA7A3 & UU E & 299 \\
\hline
\end{tabular}

Primer pairs used to amplify and differentiate serovars of $U$. parvum and subtypes of $U$. urealyticum are shown in Table 2. PCR products $(12.5 \mu \mathrm{l})$ were analysed by electrophoresis on $2.0 \%$ agarose gels, which were stained with $0.5 \mu \mathrm{g}$ ethidium bromide $\mathrm{ml}^{-1}$. For sequencing, PCR products of appropriate size that produced visible bands on UV illumination were further purified. For identification of individual sub- 


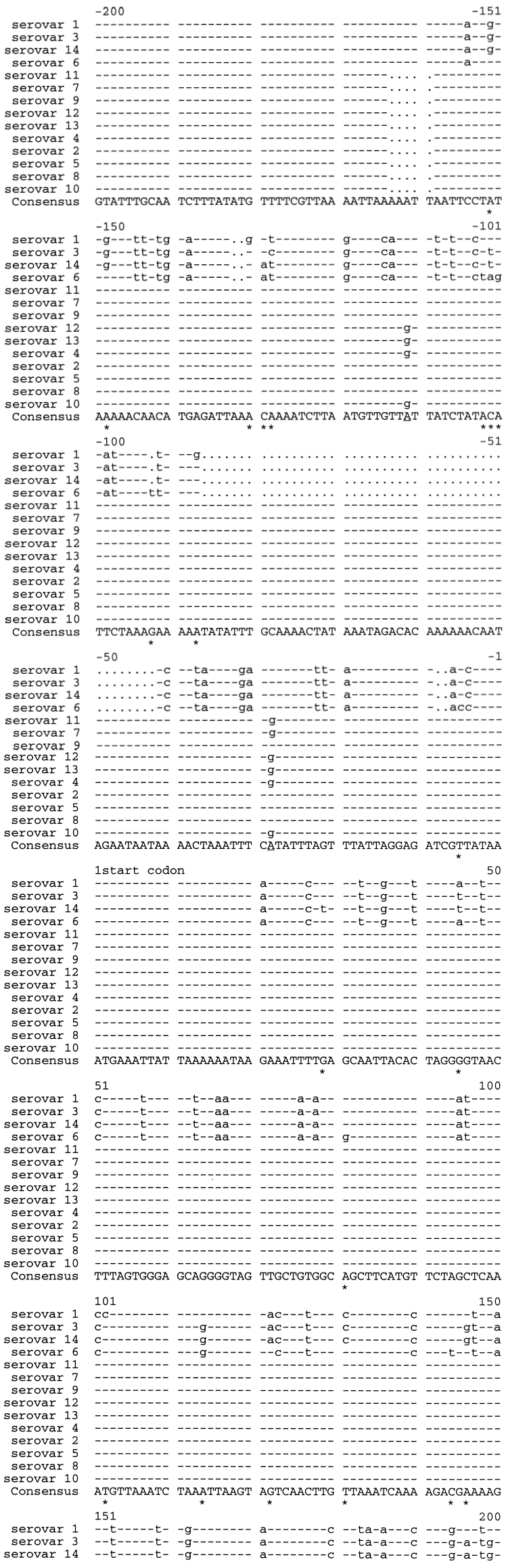

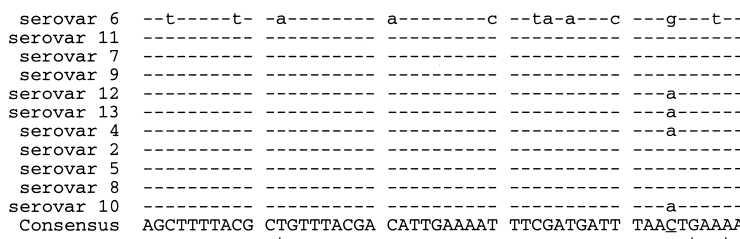

Consensus AGCTTTTACG CTGTTTACGA CATTGAAAAT TTCGATGATT TAACTGAAAA 201

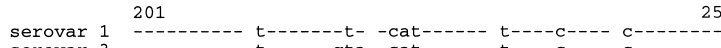

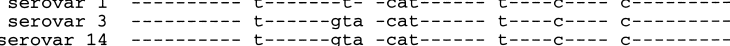

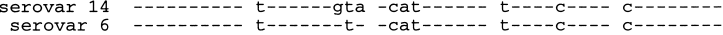
serovar 11

serovar serovar serovar 12

serovar 13

serovar

serovar 8

Consensus TGATAAAAAa GCATTAAaCG AagCTGAatT CAATGTTGCA AtTACATCAG 251

300 serovar 3 - 3 serovar 14 ------c-- -------gt a--cttga-- -------t-- -g--ggtg-

serovar 6 t------c-- --------t ct-gtt---- ------t-- gg--ggtg--

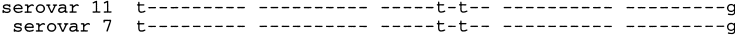

serovar 12

serovar

serovar 2

serovar

serovar 8

Consensus

CTGAAAATAA AaCAGAAAAC GCAACAACAA AagGTCACTT ACTTAACAAA 301

350

serovar

serovar 3

serovar 6

serovar 11

serovar

serovar 12

serovar 13

serovar 4

serovar

serovar 10

Consensus AAAATCTATG TTAAATTACC ACGTGAACCA AAAGCTAAAG ABCAATTAAC

351

serovar 1 - 10

serovar 14

serovar

serovar 11

serovar 7

serovar 9

serovar 12

serovar

serovar

serovar

serovar 10
Consensus

TATTATTAAT AAAGGTGGCT TACTAAAAAC TGCATCTITA GTATTACCTG 401

serovar 1

serovar

serovar

serovar 11

serovar

serovar 12

serovar 13

serovar

serovar

$\begin{array}{ll}\text { serovar } & 5 \\ \text { serovar } 8 & 8\end{array}$

serovar 10

Serovar 10

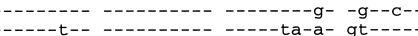
AATTTGAA TTATCAAACA GAAAAAGT=G ACTTTGAAC AA-ACAGGA 451

serovar

serovar 3

serovar 14
serovar 6

serovar

serovar 11

serovar 9

serovar 12

serovar

serovar

serovar

serovar 10

Consensus

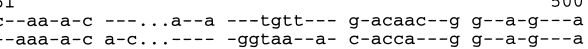

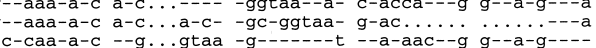
c-caa-a-c --g...gtaa $-g-------t$ t $--a-a a c--g$ g--a-g---d cca-aacc-- -gccaa-tc- --c---t-c- ccaaaa-a-g a a--...--gccaac-cc-g a-ccta-tc- --c---tac- ccaaaa-a-g a--....--ga----ac-- ---g-c---a --c-----c --t---a--- -a-gc-c-ga -a----ac-- ---g-c---a ---1-----c - - t---a--- -a-gc-c-ga -

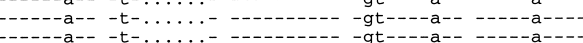
GTGGTG-AA CAA-C-CAGC AAAACCAGGA $-\mathrm{gt}-\mathrm{-t}-\mathrm{t}-\mathrm{-}-\mathrm{-}-\mathrm{-}-\mathrm{c}-\mathrm{c}$

501

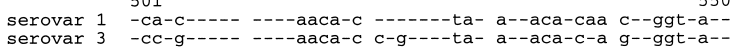

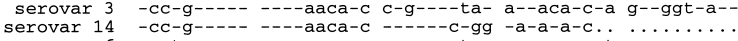

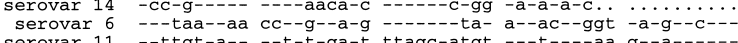
serovar 11 --ttgt-a-- --t-t-ga-t ttagc-atgt ---t----aa g--a-----

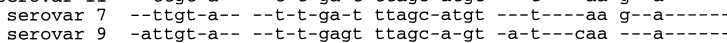




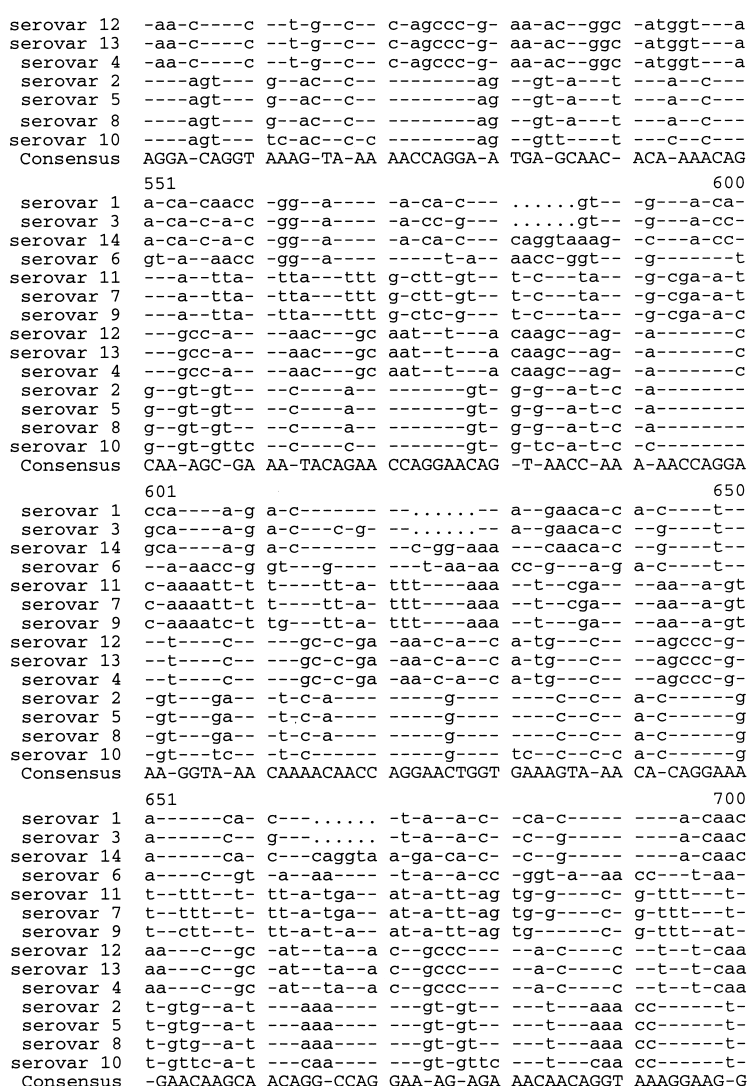

serovar 12 -aa-c----c --t-g--c-- c-agccc-g- aa-ac--ggc -atggt---a

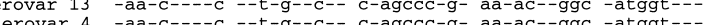
....... gt-a--aacc -gg--a---- --c-t-a-- aacc-ggt-- -g------t - a--tta- -tta---ttt g-ctt-gt-- t-c---ta-- -g-cga-a-t

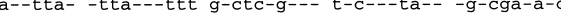
a-gaaca-c - - g----tgca--a-g a-c-c--- --c-gg-aaa ---caaca-c --g---tc-aaaatt-t t----tt-a- ttt----aaa --t--cga-- --aa--a-gt 作 -t---c-- ---gc-c-ga -aa-c-a--c a-tg---c-- --agccc-g-gt---ga-- -t-c-a---- -----g---- ----c--c-- a-c------g AA-GGTA-AA CAAAACAACC AGGAACTGGT GAAAGTA-AA CA-CAGGAAA 651 -GAACAAGCA ACAGG-CCAG GAA-AG-AGA AACAACAGGT AAAGGAAG-

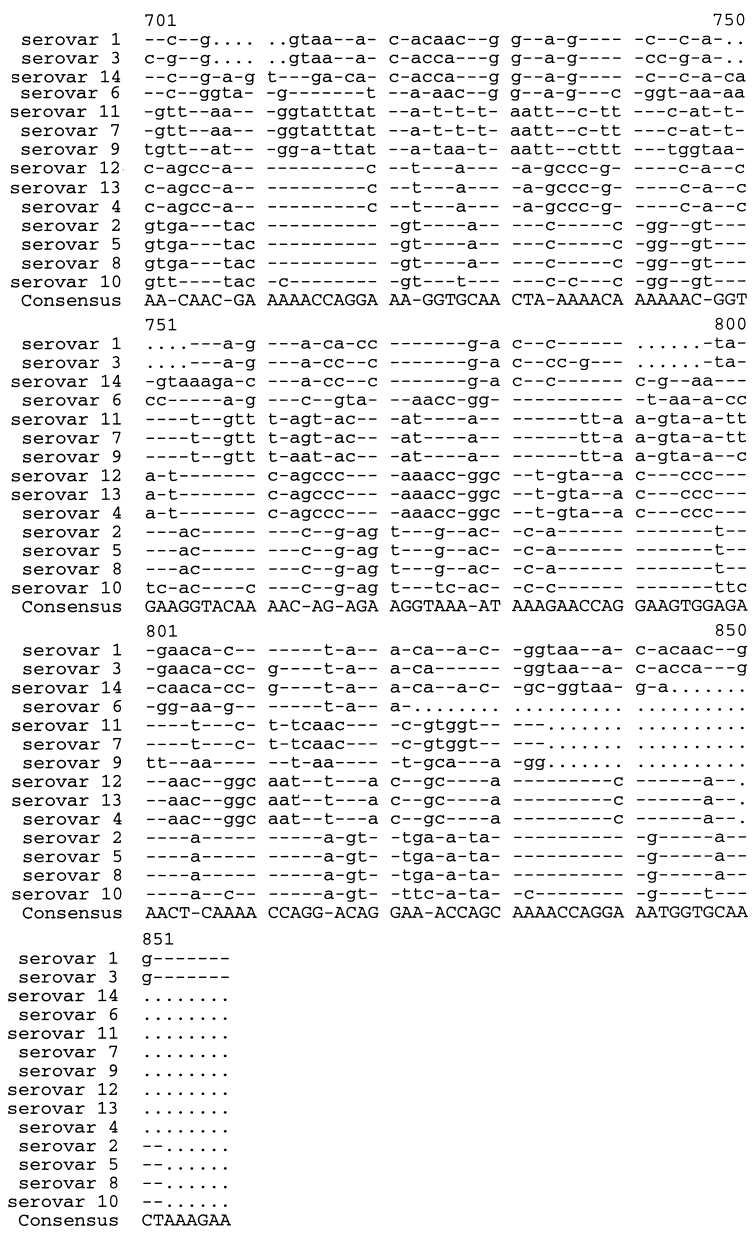

\section{a}

.

(n)

(1)

.

.

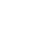

(1)

(1)

.

Fig. 1. Multiple sequence alignment of the MBA gene DNA sequences of 14 serovars of $U$. parvum and $U$. urealyticum (ATCC strains). * 45 sites of nucleotide differences between $U$. parvum serovars; underlining indicates 22 sites of nucleotide differences between $U$. urealyticum serovars.

types, the presence of PCR amplicons of expected length on ultraviolet transillumination were accepted as positive.

Sequencing and phylogenetic analysis. The PCR products of the 5'-end and the repetitive regions of the MBA genes of all the 14 ureaplasma serovars were sequenced using Applied Biosystems (ABI) Taq DyeDexoy terminator cyclesequencing kits according to standard protocols. Primers UMSPS1 (or UMSPS2) were used as sequencing primers for the amplicons of UMS-57/UMA1213 (for serovars 3 and 14), and UMS-57/UMAUA (for serovars 1 and 6); UMSUS, UMSUS1 (or UMSUS2) were used as sequencing primers for the amplicons of UMS-61/UMAUA, and UMSUS/UMAUA2 (or UMSUS/UMAUA1).

The amino acid sequences were derived by converting nucleotide sequences using Translate program from the Readseq program groups provided in WebANGIS, ANGIS (Australian National Genomic Information Service), 3rd version (mycoplasma translation codes were used). Multiple sequence alignments were performed using PILEUP and PRETTY programs from the Multiple Sequence Analysis program group, provided in WebANGIS, ANGIS (Australian National Genomic Information Service), 3rd version.
Phylogenetic relationships based on the MBA gene DNA sequences for the 14 serovars of $U$. parvum and $U$. urealyticum (ATCC strains) were studied using CLUSTAL for alignment and PHYLIP to construct the phylogenetic tree. The tree was formed using Chlamydia trachomatis (GenBank accession no. AE001315) as outgroup and was bootstrapped with 100 replications.

Nucleotide sequence accession numbers. The sequence data used in the paper are in GenBank/EMBL/DDBJ with the following accession numbers: AF056982, AF056983, AF056984; AF055358, AF055359, AF055360, AF055361, AF055362, AF055363, AF055364, AF055365, AF055366, AF055367 (Kong et al., 1999a, b).

\section{RESULTS}

\section{PCR and sequencing}

As predicted, the inner primer pair UMS$57 /$ UMA1213 produced amplicons only from serovars 3 and 14 and UMS-57/UMAUA produced amplicons from all four serovars of $U$. parvum. From $U$. urealy- 
ticum, inner primers UMS-61/UMAUA amplified portions of all 10 serovars whereas UMSUS/ UMAUA2 (or UMSUS/UMAUA1) amplified portions of seven (all except serovars 9, 7 and 11).

\section{Comparative study of the nucleotide sequences and amino acid sequences of the 5 '-end region and partial repetitive regions of MBA genes}

There were base differences at $45(45 / 601=7 \cdot 5 \%)$ sites at the 5'-end of MBA genes (-200-450) among the four serovars of $U$. parvum (Kong et al., 1999b) (Fig. 1). There were amino acids differences at 19 $(19 / 150=12.6 \%)$ sites at the N-terminus of MBA (1-159) among the four serovars of U. parvum (Fig. 2). There were base differences at $22(22 / 634=3 \cdot 5 \%)$ sites at the 5'-end of MBA genes (-200-439) among the 10 serovars of $U$. urealyticum (Fig. 1) and amino acid differences at $9(9 / 146=6 \cdot 2 \%)$ sites at the $\mathrm{N}$ terminus of MBA (1-146) (Fig. 2).

Nucleotide and amino acid sequences of the MBA gene repetitive units of $U$. parvum and $U$. urealyticum are shown in Table 3. They begin in the vicinity of nucleotide 451 (Fig. 1) and amino acid 151 (Fig. 2). There were differences between sequences from all $4 U$. parvum serovars. Sequences from serovars 2, 5 and 8 were identical and grouped as MBA genotype A. The serovar 10 sequence was the same length but differed from MBA genotype A by $3 / 24$ nucleotide bases and $2 / 8$ amino acids; this serovar was classified as MBA genotype B. Serovar 4, 12 and 13 sequences were longer than those of MBA genotypes $\mathrm{A}$ and $\mathrm{B}$, but identical with each other and were grouped together as MBA genotype C. No repetitive units were identified for serovars 9, 7 and 11 (Table 3). However, there were differences between serovar 9 and serovars $7 / 11$ in 58 $(58 / 391=14 \cdot 8 \%)$ nucleotide bases in the region $440-833$ and $14(14 / 634=2 \cdot 2 \%)$ in the region $-200-439$ at the $5^{\prime}$-end of MBA genes (Fig. 1). There were corresponding differences between serovar 9 and serovars $7 / 11$ in 31 amino acids $(31 / 130=23.8 \%)$ in the regions of $151-283$ and $6(6 / 146=4 \cdot 1 \%)$ in the region 1-146 at the N-terminus of MBA (Fig. 2). These differences defined two additional MBA genotypes, D (serovar 9) and $\mathrm{E}$ (serovars 7 and 11).

\section{The phylogenetic trees of the 14 serovars of $U$. parvum and $U$. urealyticum}

Phylogenetic tree, based on the nucleotide sequences of the $5^{\prime}$-ends and partial repetitive regions of MBA genes is shown in Fig. 3. Serovars 3 and 14 of $U$. parvum are most closely related, with serovars 1 and 6 more distant. The 10 serovars of $U$. urealyticum form five MBA genotypes as outlined above. MBA genotypes $\mathrm{A}, \mathrm{C}$ and $\mathrm{E}$ are separate clusters of three, three and two serovars, respectively, with the MBA genotype $\mathrm{B}$ (a single serovar) located between MBA genotypes A and C; MBA genotype D (also a single serovar) is located between MBA genotypes C and E (Fig. 3).

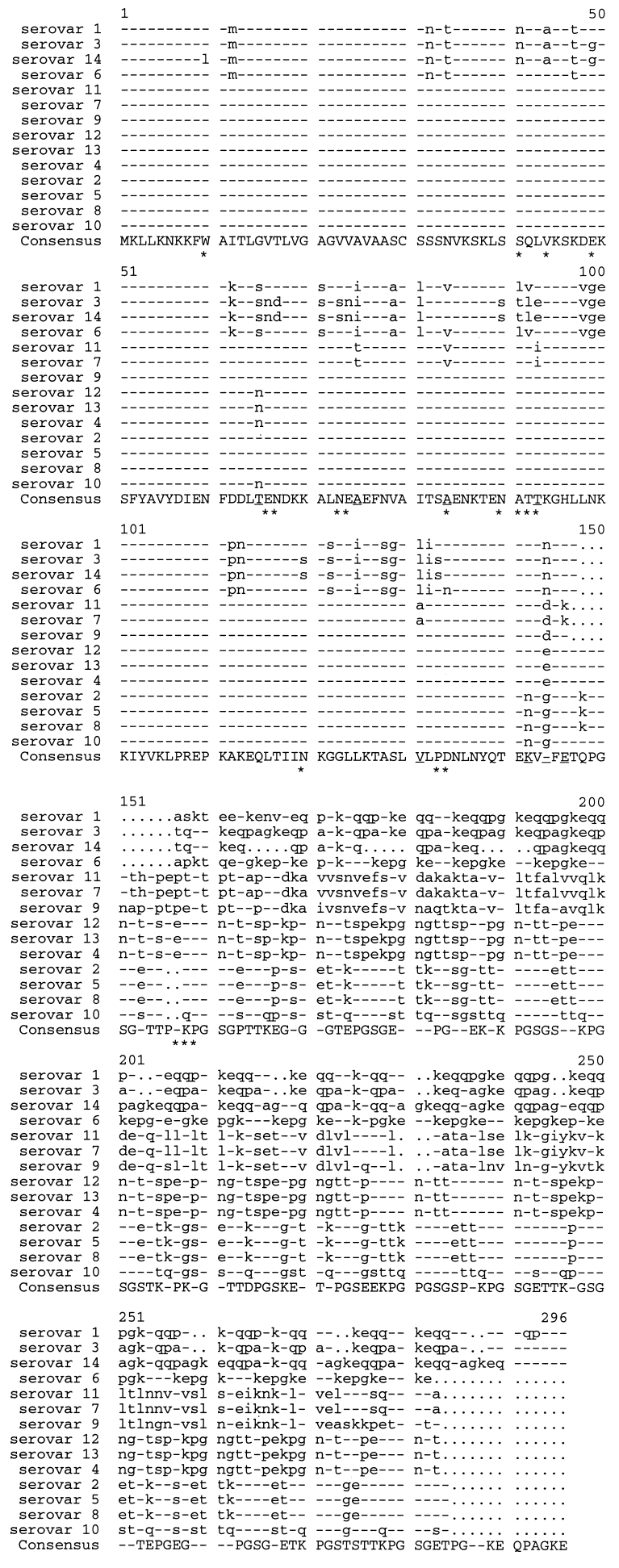

Fig. 2. Multiple sequence alignment of the MBA amino acid sequences of 14 serovars of $U$. parvum and $U$. urealyticum (ATCC strains). *19 sites of amino acid differences between $U$. parvum serovars; underlining indicates nine sites of amino acid differences between $U$. urealyticum serovars.

\section{The specificity of $U$. urealyticum subtyping primers}

All the ATCC and UAB reference strains of $U$. parvum and $U$. urealyticum were correctly identified by the 
Table 3. Nucleotide and amino acid sequences of repetitive units of the MBA genes of 14 serovars of $U$. parvum and U. urealyticum

UP: U. parvum; UU: U. urealyticum. UU MBA genotype A: includes serovars 2, 5 and 8; UU MBA genotype B: includes serovar 10; UU MBA genotype C: includes serovars 4, 12 and 13; UU MBA genotype D: includes serovar 9; UU MBA genotype E: includes serovars 7 and 11 .

\begin{tabular}{|llc|}
\hline Serovar/subtype & Nucleotide sequence & $\begin{array}{c}\text { Amino acid } \\
\text { sequence }\end{array}$ \\
\hline UP Serovar 1 & CAA CAA CCA GGT AAA GAA & QQPGKE \\
UP Serovar 3 & CAA CCA GCA GGT AAA GAA & QPAGKE \\
UP Serovar 6 & GGT AAA GAA CCA & PGKE \\
UP Serovar 14 & CAA CAA CCA GCA GGT AAA GAA QPAGKE \\
UU MBA genotype A & ACA AAA CCA GGA AGT GGT GAA ACT & TKPGSGET \\
UU MBA genotype B & ACA CAA CCA GGA AGT GGT TCA ACT & TQPGSGST \\
UU MBA genotype C & ACA AGC CCA GAA AAA CCA GGC AAT GGT ACA & TSPEKPGNGT \\
UU MBA genotype D & - & - \\
UU MBA genotype E & - & - \\
\hline
\end{tabular}

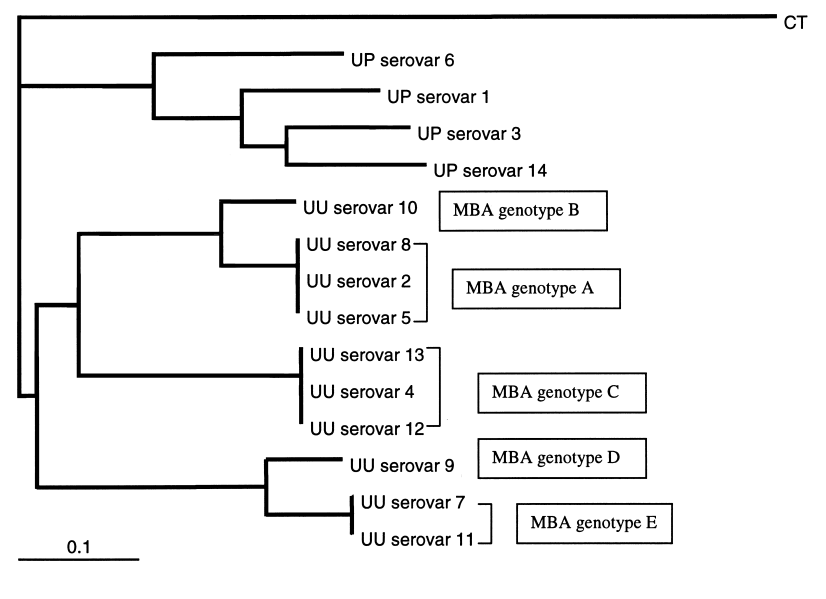

Fig. 3. Phylogenetic tree for 14 serovars of U. parvum (UP) and $U$. urealyticum (UU) (ATCC strains) based on the MBA gene DNA sequences. CLUSTAL was used for alignment, and PHYLIP was used for constructing the phylogenetic tree. The tree was formed using Chlamydia trachomatis (GenBank no. AE001315) as outgroup and was bootstrapped with 100 replications.

serovar-/MBA genotype-specific primers. The results of PCR for all the serovars of $U$. parvum and $U$. urealyticum, using the 33 subtype-specific primer pairs to amplify the 5'-end of the MBA genes are summarized in Table 2 and representative examples are shown as Figs 4 and 5.

Our primary evaluation showed that the serovars and MBA genotypes (corresponding with serovars) of $U$. parvum and $U$. urealyticum were identified, specifically, using primer pairs as shown in Table 2.

\section{DISCUSSION}

Various phenotypic and molecular methods have been described previously to distinguish the two main groups of human ureaplasmas (formerly two biovars of $U$. urealyticum, now proposed species $U$. parvum

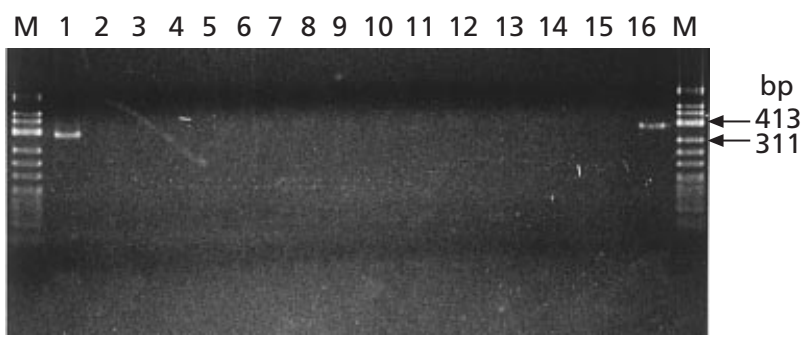

Fig. 4. Results of PCR amplification of the $5^{\prime}$-end of MBA genes of all 14 serovars of $U$. parvum and $U$. urealyticum using primers UMS-83 and UMA269'. U. parvum consists of serovars 1 , 3,6 and $14 ; U$. urealyticum consists of serovars $2,4,5,7,8,9$, 10, 11, 12 and 13. Lanes: $M$, molecular mass markers $\phi X 174$ DNA/Hinfl; 1 and 16, $U$. parvum serovar 1 ATCC strain and UAB reference strain; 6 and 7, U. parvum serovar 6 ATCC strain and $U A B$ reference strain; $2-5, U$. urealyticum serovar $2, U$. parvum serovar 3, U. urealyticum serovars 4 and 5 ATCC strains; 8-15, $U$. urealyticum serovars $7-13$ and $U$. parvum serovar 14 ATCC strains.

and $U$. urealyticum) (previously summarized by Kong et al., 1999b). Our previous study showed that homology between sequences of the 16S rRNA genes, $16 \mathrm{~S}-23 \mathrm{~S}$ rRNA intergenic spacer regions and urease gene subunits of serovars within each proposed species was high and these regions could not be used for further subtyping (Kong et al., 1999b, 2000). However, sequence differences between the partial $5^{\prime}$-end regions of the MBA genes allowed each species to be divided into three genotypes (Kong et al., 2000). It had been suggested previously that the repetitive region of the MBA gene should also contain serovar-specific definition sites (Zheng et al., 1996). Therefore, in this study, we sequenced the whole 5 -end regions of the MBA genes of the 10 serovars of $U$. urealyticum and partial repetitive regions of the MBA genes for all 14 ureaplasma serovars. Our aim was to define sequence differences that would allow further molecular identi- 


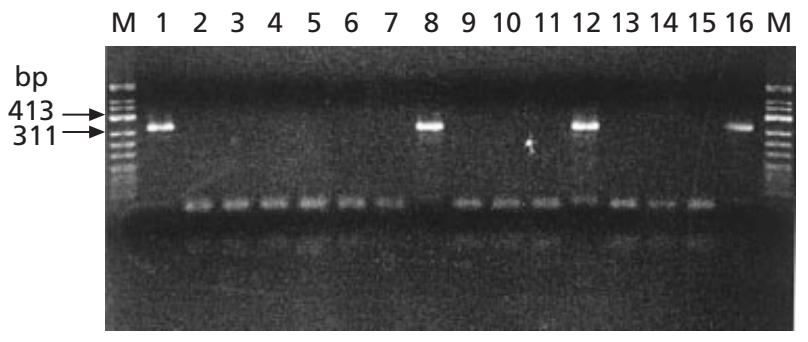

Fig. 5. Results of PCR amplification of the $5^{\prime}$-end of MBA genes of all 14 serovars of $U$. parvum and $U$. urealyticum using primers UMS-61 and UMA7A1. U. parvum consists of serovars 1 , 3,6 and $14 ; U$. urealyticum consists of serovars $2,4,5,7,8,9$, $10,11,12$ and 13. Lanes: $M$, molecular mass markers $\phi \times 174$ DNA/Hinfl; 1 and $8, U$. urealyticum serovar 7 UAB reference strain and ATCC strain; 12 and 16, U. urealyticum serovar 11 ATCC strain and UAB reference strain; 2-7, U. parvum serovar 1 , $U$. urealyticum serovar $2, U$. parvum serovar $3, U$. urealyticum serovars 4, 5 and $U$. parvum serovar 6 ATCC strains; 9-11, $U$. urealyticum serovars 8,9 and 10 ATCC strains; 13-15, U. urealyticum serovars 12,13 and $U$. parvum serovar 14 ATCC strains.

fication of serovars or additional MBA genotypes of $U$. parvum and $U$. urealyticum (Kong et al., 2000).

Our previous studies showed only three base differences between sequences of the 5'-end of MBA gene of U. parvum serovars 3 and 14 (Kong et al., 1999a, b). In this study we showed more numerous differences in nucleotide and amino acid sequences of the repetitive units, between $U$. parvum serovars, which allowed all of them, including serovars 3 and 14, to be differentiated. Based on our previous study of partial 5'end sequences of the MBA genes of $U$. urealyticum (Kong et al., 1999b), serovar 10 is closely related to serovars 4, 12 and 13. However, differences in nucleotide and amino acid sequences immediately upstream of the repetitive regions and in the repetitive units themselves, allowed serovar 10 to be separated from serovars $4 / 12 / 13$. Similarly, serovar 9 was closely related to serovars 2,5 and 8 , based on sequences of the 5'-end of the MBA genes (Kong et al., 1999b) but deletion of the repetitive region in serovar 9 allowed it to be differentiated from serovars $2 / 5 / 8$. This finding is supported by the recent development of a monoclonal antibody against $U$. urealyticum serovar 9 , that cross-reacts minimally only with serovar 2 (Naessens et al., 1998a).

The present study also showed that there were 22 bases at the $5^{\prime}$-end of the MBA genes of the 10 serovars of $U$. urealyticum, upstream of the repetitive regions, which helped to differentiate the five MBA genotypes. More than half of these differences were between MBA genotype E (serovars 7/11) and the other four MBA genotypes. Serovars $7 / 11$ were similar to serovar 9 in that the repetitive sequences were deleted. However, their sequences differed by 14 bases at the $5^{\prime}$-end of MBA genes and 58 within sequences that corresponded with those of the repetitive regions of MBA genes of the other serovars (Fig. 1). Serovars in MBA genotypes
A (serovars 2, 5 and 8), C (serovars 4, 12 and 13) and E (serovars 7 and 11) could not be differentiated further on the basis of these sequences.

Reliable differentiation between the serovars of $U$. urealyticum using phenotypic methods is difficult. Antigenic cross-reactions between serovars 2 and 5 (Wiley \& Quinn, 1984; Quinn et al., 1981), 4 and 8 (Quinn et al., 1981), and 8, 2 and 4 (Robertson \& Stemke, 1979) have been described. Because of variable strain selection from frequently mixed cultures, the reproducibility of serovar determination between primary and secondary plating of isolates was only $83 \%$; it increased only to $87 \%$ on multiple, secondary cultures (Stemke \& Roberston, 1985). The fact that genetic differences between some serovars are minor has been confirmed by demonstration that a single amino acid difference between the MBA of serovars 3 and 14 of $U$. parvum accounts for epitope differences that can be distinguished using type-specific monoclonal anibodies (Zheng et al., 1996). Arbitrarily primed PCR, using a pairwise combination of primers, was able to differentiate only a few of the 10 serovars of $U$. urealyticum (Grattard et al., 1995).

Further work is required to identify other genes or other regions of the MBA genes that may be used to differentiate $U$. urealyticum serovars within MBA genotypes A, C and E. However, on the basis of our data, we suggest that genetic and antigenic differences between some serovars are so minor that further subdivision into serovars might be artificial and/or unnecessary. These data provide a better understanding of the molecular basis of serotype differentiation. Based on the phylogenetic analysis, we designed a series of serovar-/MBA genotype-specific primer pairs to subtype each Ureaplasma species and are developing a more practical subtyping system that can be used for further study of the relationship between subtypes and diseases.

\section{ACKNOWLEDGEMENTS}

We thank Mark Wheeler for assistance with sequencing.

\section{REFERENCES}

Abele-Horn, M., Wolff, C., Dressel, P., Pfaff, F. \& Zimmermann, A. (1997). Association of Ureaplasma urealyticum biovars with clinical outcome for neonates, obstetric patients, and gynaecological patients with pelvic inflammatory disease, J Clin Microbiol 35, 1199-1202.

Cracea, E., Constantinescu, S. \& Lazar, M. (1985). Serotypes of Ureaplasma urealyticum isolated from patients with nongonococcal urethritis and gonorrhea and from asymptomatic urethral carriers, Sex Transm Dis 12, 219-223.

Grattard, F., Pozzetto, B., de Barbeyrac, B., Renaudin, H., Clerc, M., Gaudin, O. G. \& Bebear, C. (1995). Arbitrarily-primed PCR confirms the differentiation of strains of Ureaplasma urealyticum into two biovars, Mol Cell Probes 9, 383-389. 
Hannaford, K., Todd, D. A., Jeffrey, H., John, E., Byth, K. \& Gilbert, G. L. (1999). The role of Ureaplasma urealyticum in lung disease of prematurity, Arch Dis Child Fetal Neonatal Ed 81, F162-167.

Hewish, M. J., Birch, D. F. \& Fairley, K. F. (1986). Ureaplasma urealyticum serotypes in urinary tract disease, J Clin Microbiol 23, 149-154.

Kong, F., Zhu, X., Wang, W., Zhou, X., Gordon, S. \& Gilbert, G. L. (1999a). Comparative analysis and serovar-specific identification of the multiple banded antigen genes of Ureaplasma urealyticum biovar one, J Clin Microbiol 37, 538-543.

Kong, F., James, G., Ma, Z., Gordon, S., Wang, B. \& Gilbert, G. L. (1999b). Phylogenetic analysis of Ureaplasma urealyticum support for the establishment of a new species, Ureaplasma parvum, Int J Syst Bacteriol 49, 1879-1889.

Kong, F., Ma, Z., James, G., Gordon, S. \& Gilbert, G. L. (2000). Species identification and subtyping of Ureaplasma parvum and Ureaplasma urealyticum using PCR-based assays, J Clin Microbiol 38, 1175-1179.

Kundsin, R. B., Leviton, A., Allred, E. N. \& Poulin, S. A. (1996). Ureaplasma urealyticum infection of the placenta in pregnancies that ended prematurely, Obstet Gynecol 87, 122-127.

Naessens, A., Foulon, W., Breynaert, J. \& Lauwers, S. (1988). Serotypes of Ureaplasma urealyticum isolated from normal pregnant women and patients with pregnancy complications, J Clin Microbiol 26, 319-322.

Naessens, A., Cheng, X., Lauwers, S. \& Robertson, J. A. (1998). Development of a monoclonal antibody to a Ureaplasma urealyticum serotype 9 antigen, J Clin Microbiol 36, 1125-1127.

Quinn, P. A., Arshoff, L. U. \& Li, H. C. (1981). Serotyping of Ureaplasma urealyticum by immunoperoxidase assay, $J$ Clin Microbiol 13, 670-676.

Razin, S. \& Yogev, D. (1986). Genetic relatedness among Ureaplasma urealyticum serotypes (serovars), Pediatr Infect Dis 5 (suppl. 6), S300-S304.

Robertson, J. A. \& Stemke, G. W. (1979). Modified metabolic inhibition test for serotyping strains of Ureaplasma urealyticum (T-strain Mycoplasma), J Clin Microbiol 9, 673-676.
Robertson, J. A. \& Stemke, G. W. (1982). Expanded serotyping scheme for Ureaplasma urealyticum strains isolated from humans, J Clin Microbiol 15, 873-878.

Robertson, J. A., Honore, L. H. \& Stemke, G. W. (1986). Serotypes of Ureaplasma urealyticum in spontaneous abortion, Pediatr Infect Dis 5 (suppl. 6), S270-S272.

Stemke, G. W. \& Robertson, J. A. (1985). Problems associated with serotyping strains of Ureaplasma urealyticum, Diagn Microbiol Infect Dis 3, 311-320.

Taylor-Robinson, D., Furr, P. M. \& Webster, A. D. (1985). Ureaplasma urealyticum causing persistent urethritis in a patient with hypogammaglobulinaemia, Genitourin Med 61, 404-408.

Teng, L. J., Ho, S. W., Ho, H. N., Liaw, S. J., Lai, H. C. \& Luh, K. T. (1995). Rapid detection and biovar differentiation of Ureaplasma urealyticum in clinical specimens by PCR, J Formos Med Assoc 94, 396-400.

Viarengo, J., Hebrant, F. \& Piot, P. (1980). Ureaplasma urealyticum in the urethra of healthy men, Br J Vener Dis 56, 169-172.

Watson, H. L., Blalock, D. K. \& Cassell, G. H. (1990). Variable antigens of Ureaplasma urealyticum containing both serovarspecific and serovar-cross-reactive epitopes, Infect Immun 58, 3679-3688.

Wiley, C. A. \& Quinn, P. A. (1984). Enzyme-linked immunosorbent assay for detection of specific antibodies to Ureaplasma urealyticum serotypes, J Clin Microbiol 19, 421-426.

Zheng, X., Watson, H. L., Waites, K. B. \& Cassell, G. H. (1992). Serotype diversity and antigen variation among invasive isolates of Ureaplasma urealyticum from neonates, Infect Immun 60, 3472-3474.

Zheng, X., Teng, L. J., Watson, H. L., Glass, J. I., Blanchard, A. \& Cassell, G. H. (1995). Small repeating units within the Ureaplasma urealyticum $\mathrm{MB}$ antigen gene encode serovar specificity and are associated with antigen size variation, Infect Immun 63, 891-898.

Zheng, X., Lau, K., Frazier, M., Cassell, G. H. \& Watson, H. L. (1996). Epitope mapping of the variable repetitive region with the MB antigen of Ureaplasma urealyticum, Clin Diagn Lab Immunol 3, 774-778. 